\begin{tabular}{c} 
International Journal of Biological Research, 3(1)(2015) 9-11 \\
International Journal of Biological Research \\
SPC \\
Journal home page: $\begin{array}{c}\text { www.sciencepubco.com/index.php/IJBR } \\
\text { doi: } 10.14419 / i j b r . v 3 i 1.3770 \\
\text { Research Paper }\end{array}$ \\
\hline
\end{tabular}

\title{
Anatomical studies on two species of Dracaena in southeastern Nigeria
}

\author{
Chinyere .V. Ilodibia ${ }^{1}{ }^{*}$, Rachael. U. Ugwu ${ }^{1}$, C. U. Okeke ${ }^{1}$,Ebele E. Akachukwu ${ }^{2}$, \\ Bibian O. Aziagba ${ }^{1}$, Nneka F. Okeke ${ }^{1}$ \\ ${ }^{I}$ Department of Botany, Nnamdi Azikiwe University, P. M. B. 5025 Awka, Anambra State, Nigeria \\ ${ }^{2}$ Department of Biology, Nwafor Orizu College of Education Nsugbe, Anambra State, Nigeria \\ *Corresponding author E-mail: chinyereokafor206@yahoo.com
}

\begin{abstract}
Anatomical studies of transverse, tangential and radial longitudinal sections of roots, stems and leaves of two species of Dracaena present in southeastern Nigeria were investigated, to determine their anatomical features with regards to arrangement of vascular bundles in their roots, stems and leaves and type of secondary growth occurring in them using standard method. T- test was used for the analyses. Result showed that the number of vascular bundles in the leaves and roots of $D$. arborea are more in number than those of $D$. mannii but the reverse is the case in the stem. Secondary growth occurs in both D. mannii and D. arborea. The overall analyses showed no significant statistical difference in the anatomy of the two Dracaena species. The implication is that the two species are closely related and this justified their placement under the same genus Dracaena while the slight differences between them suggest the reason for their separation into different species.
\end{abstract}

Keywords: Anatomy; Dracaena Arborea; Dracaena Mannii; Radial Section; Secondary Growth; Tangential Section; Transverse Section; Vascular Bundle.

\section{Introduction}

The name Dracaena L. is derived from the Greek word drakaina which means a "dragon" and alludes the sap of the stems of Dracaena draco, which was said to resemble dragon's blood. The genus, Dracaenas belongs to the family Agavaceae which includes about 550-600 species in around 18 genera (Pires et al, 2004). The genus was first described by Linnaeus in 1767. Some species of Dracaena include Dracaena fragrans, D.surculosa, D. draco, D. marginata, D. arborea, D goldiana., D. sanderina, D. deremensis, D. reflexa, D. mannii etc. Dracaenas are either shrubs or trees and divided into two broad groups based on their growth habits- tree Dracaenas and shrubby Dracaenas. Tree Dracaenas include Dracaena Americana(Central American dragon tree), D. draco (Canary Islands dragon tree), D. marginata, D. mannii etc while shrubby Dracaenas include D. aletriformis, D. bicolar, D. cincta, D. concinna, etc(Waterhouse, 1987). Dracaenas are used as ornamentals, medicinal plants, in photo engraving, in research, as hedge plants, colourants etc. In Europe and Canada they are cultivated and sold as ornamentals (Huxley, 1992). Dracaena mannii Bak. and Dracaena arborea Link.which are the commonly ones in southeastern Nigeria are the point of interest in this study. To classify plants, taxonomists make use of anatomy, morphology etc as taxonomic lines of evidence to determine their similarities and differences in order to group them into various taxa.

Anatomy is essentially of great importance in plant taxonomy. Pandey (2004), defined anatomy as the "study of gross internal structure of plant organs by the technique of section cutting". In discussing anatomy in relation to taxonomy, he said that anatomy is of restricted value for distinguishing species or groups of less than specific rank, because the differences between them are usually quatitative rather than qualitative, and that "it is however, most important to rely upon anatomical characters of which the taxonomic value has been established".

Anatomical characters of taxonomic importance include such characters as type of stomata, width of medullary rays, presence of bicollateral bundles, wood characters etc (Pandey, 2004). The characteristics of the wood (xylem) that possess greatest taxonomic value and diagnostic features are - the vessels: the distribution, pattern diameter and frequency as seen in transverse sections, the presence of tyloses etc.

The objective of this research, therefore, was to study the anatomical features of the transverse, tangential and radial sections of Dracaena mannii and Dracaena arborea with regards to arrangement of vascular bundles in their roots, stems and leaves and type of secondary growth occurring in them.

\section{Materials and method}

\subsection{Sources of materials}

Leaves, stems, and roots, of Dracaena species were collected from Nsukka town (N060.86. 43.5 and E070.42.56.0) in Nsukka, Nsukka Local Government Area Enugu State, Nigeria.

The Dracaena Species were authenticated at Bio-diversity Development and conservation, Nsukka, where the voucher specimens were deposited. 


\subsection{Anatomical analysis}

Sectioning of leaf, stem and root of specimens was done using a sledge microtome. The sections were transferred into a staining jar and stained in safranin for $5 \mathrm{~min}$. The safranin was drained off and the sections washed three times with distilled water. The sections were washed again with 97 percent alcohol and absolute alcohol for two min. each and counter stained in one percent fast green for $5 \mathrm{~min}$. and then washed with absolute alcohol 4 times. The sections were transferred into a staining jar containing 50/50 alcohol/xylene and washed until they became very clear. Pure xylene was used to finally clear the sections in the staining dish. Canada balsam was then used to mount the specimens on slides. Each slide was carefully covered with a $22 \mathrm{~mm} \times 22 \mathrm{~mm}$ cover-slip. The mounted specimens were observed under a light microscope and photomicrographs were taken (2.0 model; China). This procedure is as outlined by Anon (1968).

\subsection{Statistical procedure}

The results were analyzed using T- test and presented in mean \pm standard deviation (SD).

\section{Result}

The results of the study were shown below. The number of vascular bundles per field view in the leaves, stems and roots of $D$. mannii and $D$. arborea calculated at different magnifications are presented in Fig. 1, 2, 3, and Tables 1,

2 and 3.

B

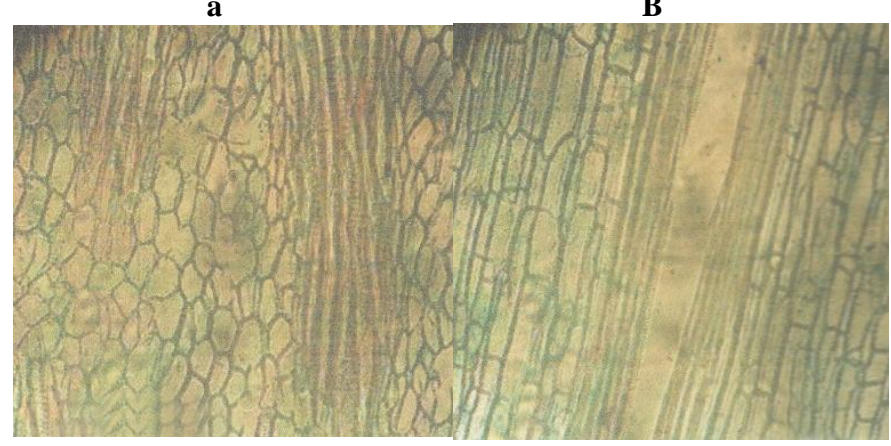

D
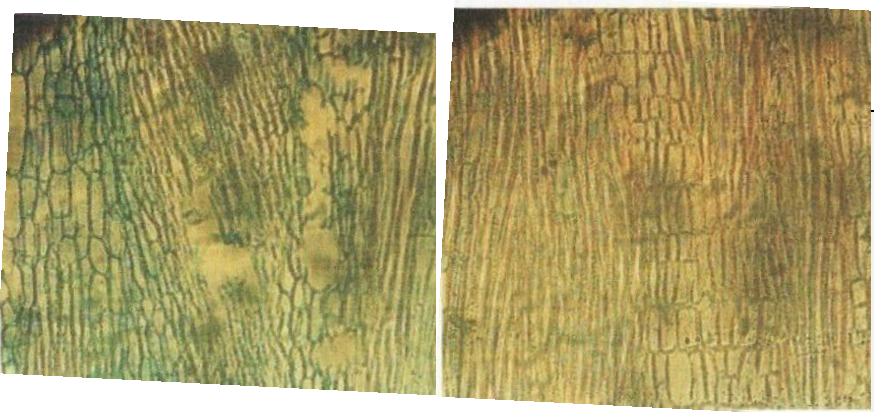

Fig. 1: (A-D) = A: TLS. Root of D. mannii, B: TLS. Root of D. arborea, C: TLS of Stem of D. mannii, D: TLS. Stem of D. arborea. (Tangential Longitudinal Section TLS).

$\mathbf{a}$

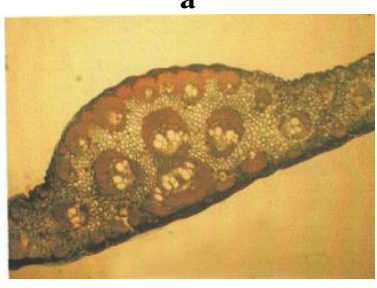

c

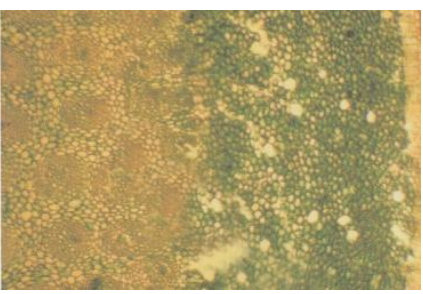

e

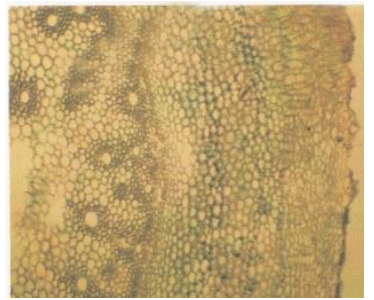

Fig. 2: $(\mathrm{A}-\mathrm{F})=\mathrm{A}$ : T/S. Leaf of D. mannii, B: T/S. Leaf of D. arborea, C: T/S of Stem of $D$. arborea, D: T/S of Stem of $D$. mannii, E: T/S of Root of D. mannii, F: T/S of Root of D.arborea (Transverse Section T/S). a

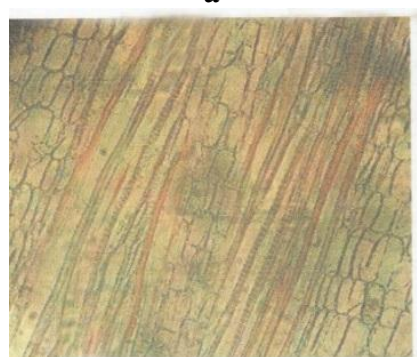

c

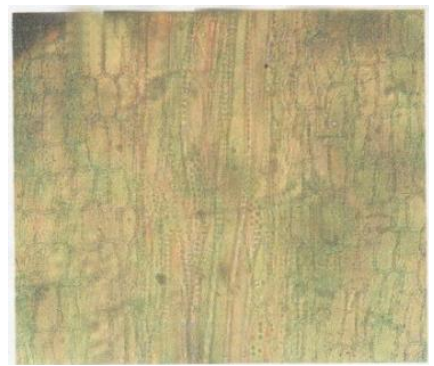

b

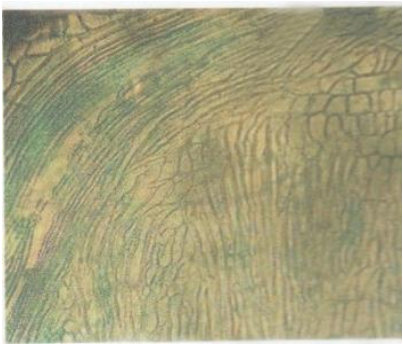

d

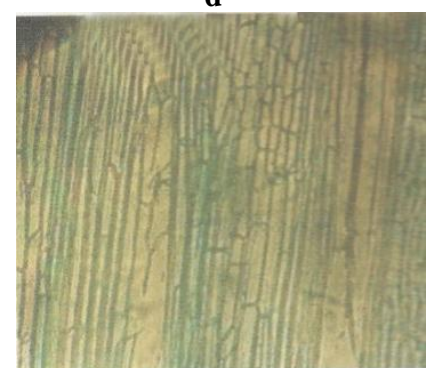

Fig. 3: (A-D) = A: RLS. Stem of D. mannii, B: RLS. Stem of D. arborea, C: RLS. Root of D. mannii, D: RLS. Root of D. arborea, (Radial Longitudinal Section RLS).

Table 1: Number of Vascular Bundle per 10 Field Views in the Leaf of $D$. mannii and D. arborea at $\mathrm{x} 100$ and $\times 400$.

\begin{tabular}{llll} 
X100 & & $\mathrm{X} 400$ & \\
D. mannii & D. arborea & D. mannii & D.arborea \\
$0.4 \pm 1.83$ & $01 \pm 4.93$ & $01 \pm 0.38$ & $01 \pm 0.66$ \\
$14 \pm 1.33$ & $03 \pm 4.30$ & $02 \pm 0.38$ & $01 \pm 0.35$ \\
$24 \pm 4.49$ & $15 \pm 0.15$ & $04 \pm 0.57$ & $05 \pm 0.60$ \\
$16 \pm 1.96$ & $38 \pm 6.77$ & $02 \pm 0.06$ & $07 \pm 1.23$ \\
$12 \pm 0.70$ & $37 \pm 6.45$ & $03 \pm 0.25$ & $03 \pm 0.03$ \\
$10 \pm 0.06$ & $23 \pm 2.02$ & $03 \pm 0.25$ & $05 \pm 0.60$ \\
$07 \pm 0.89$ & $21 \pm 1.39$ & $04 \pm 0.54$ & $03 \pm 0.03$ \\
$05 \pm 1.52$ & $17 \pm 0.13$ & $01 \pm 0.38$ & $02 \pm 0.35$ \\
$03 \pm 2.15$ & $07 \pm 3.04$ & $02 \pm 0.06$ & $02 \pm 0.35$ \\
$03 \pm 2.15$ & $04 \pm 3.98$ & $01 \pm 0.38$ & $01 \pm 0.66$ \\
\hline
\end{tabular}

The table shows that vascular bundles are more numerous in the leaf of D. arborea than in D. mannii at x100 and x400 magnification respectively. 
Table 2: Number of Vascular Bundles per 10 Field Views in the Roots of D. arborea and D. mannii. at X100 and X400 magnifications.

\begin{tabular}{llll}
\hline X100 & & X 400 & \\
D.arborea & D.mannii & D.arborea & D.mannii \\
$18 \pm 1.52$ & $17 \pm 2.09$ & $03 \pm 0.32$ & $02 \pm 0.41$ \\
$13 \pm 0.06$ & $12 \pm 0.51$ & $02 \pm 0.00$ & $02 \pm 0.41$ \\
$19 \pm 1.83$ & $12 \pm 0.51$ & $02 \pm 0.00$ & $02 \pm 0.41$ \\
$19 \pm 1.83$ & $14 \pm 1.14$ & $02 \pm 0.00$ & $01 \pm 0.22$ \\
$15 \pm 0.57$ & $15 \pm 1.45$ & $02 \pm 0.00$ & $02 \pm 0.41$ \\
$13 \pm 0.06$ & $12 \pm 0.51$ & $02 \pm 0.00$ & $02 \pm 0.41$ \\
$10 \pm 1.01$ & $07 \pm 1.08$ & $02 \pm 0.00$ & $01 \pm 0.22$ \\
$11 \pm 0.70$ & $05 \pm 1.71$ & $02 \pm 0.00$ & $01 \pm 0.22$ \\
$09 \pm 1.33$ & $06 \pm 1.39$ & $02 \pm 0.00$ & $01 \pm 0.22$ \\
$05 \pm 2.59$ & $04 \pm 2.02$ & $01 \pm 0.32$ & $01 \pm 0.22$ \\
\hline
\end{tabular}

The result on the above table shows that vascular bundles are more numerous in the root of D.arborea than in D. mannii at x100 and x400 magnifications.

Table 3: Number of Vascular Bundles per 10 Field Views in the Stems of D. arborea and D. mannii at $\mathrm{x} 40$ and $\mathrm{x} 100$ magnifications.

\begin{tabular}{llll}
\hline X40 & \multicolumn{3}{c}{ X100 } \\
D. arborea & D. mannii & D. arborea & D. mannii \\
$88 \pm 0.13$ & $102 \pm 1.04$ & $25 \pm 0.57$ & $33 \pm 1.04$ \\
$95 \pm 2.34$ & $112 \pm 4.21$ & $19 \pm 1.33$ & $38 \pm 0.54$ \\
$90 \pm 0.76$ & $128 \pm 9.27$ & $22 \pm 0.38$ & $35 \pm 0.41$ \\
$96 \pm 2.66$ & $90 \pm 2.75$ & $20 \pm 1.01$ & $43 \pm 2.11$ \\
$94 \pm 2.02$ & $115 \pm 5.15$ & $22 \pm 0.38$ & $40 \pm 1.17$ \\
$83 \pm 1.45$ & $90 \pm 2.75$ & $23 \pm 0.06$ & $37 \pm 0.22$ \\
$86 \pm 0.51$ & $100 \pm 0.41$ & $27 \pm 1.20$ & $31 \pm 1.68$ \\
$86 \pm 0.51$ & $102 \pm 1.04$ & $25 \pm 0.57$ & $35 \pm 0.41$ \\
$81 \pm 2.09$ & $70 \pm 9.08$ & $23 \pm 0.06$ & $37 \pm 0.22$ \\
$77 \pm 3.35$ & $78 \pm 6.55$ & $26 \pm 0.89$ & $34 \pm 0.73$ \\
\hline
\end{tabular}

From the table above, there are more vascular bundles in the stem of $D$. mannii than in D. arborea stem at x40 and x100 magnifications.

\section{Discussion}

The research carried out revealed that secondary growth occurs in $D$. mannii and D. arborea as in other groups of monocots. According to Waterhouse, (1987) Dracaenas have a secondary thickening meristem in their trunk which is quite different from the thickening meristem found in dicotyledonous plants and is termed "Dracaenoid thickening". This character is shared with other members of the Agavaceae and Xanthorrhoeaceae among other related families, (Waterhouse, 1987). The meristem concerned with this growth called cambium, according to Pandey, (2004) appears to be a direct continuation of a primary thickening meristem. However, the cambium functions in the part of the axis that has completed its elongation. The cambium originates in the parenchyma outside the vascular bundles. This part of the axis is sometimes identified as cortex, and sometimes as pericycle (Waterhouse, 1987). The newly formed cambium cut cells towards outside and inside both. The tissues developed on the inner side of the cambium are usually differentiated into vascular bundles and remain separated from each other by lignified tissue. This tissue, according to Pandey (2004) sometimes remains unlignified and thin-walled. The cells formed on the outer side of the cambium make parenchyma. The cross-section of Dracaena stem showed the singled-layered epidermis which remained covered with thick cuticle. The lenticels were also seen in the epidermis. Beneath the epidermis hypodermis, the cork arises which gives rise to the cork towards outside. Below the cork cambium, well developed parenchyma is present.

With regards to distribution of vascular bundles in the leaves, stems and roots of $D$. mannii and $D$. arborea, the results showed that vascular bundles were more numerous in the leaf of $D$. arborea $(38 \pm 6.77$ and $07 \pm 1.23)$ than in D. mannii $(24 \pm 4.49$ and $04 \pm 0.57)$ at $x 100$ and $x 400$ magnifications respectively (Table1). The root of $D$. arborea had more vascular bundles $(19 \pm 1.83$ and $03 \pm 0.32)$ than that of $D$. mannii $(17 \pm 2.09$ and $02 \pm 0.41)$ at $x 100$ and $\mathrm{x} 400$ magnifications respectively (Table 2 ) while there were more vascular bundles $(128 \pm 9.27$ and $43 \pm 2.11)$ in the stem of $D$. mannii than in D. arborea stem $(96 \pm 2.66$ and $27 \pm 1.20)$ at $\mathrm{x} 40$ and x100 magnifications respectively (Table 3 ). There was however, no significant difference in the anatomy of the two species of Dracaena at $\mathrm{p}=0.05$.

\section{Conclusion}

The result of the study showed that there is no significant difference in the anatomy of $D$. mannii and $D$. arborea. In other words, the two species are closely related and this justifies their placement in the same genus Dracaena. The slight differences existing between them also justify their separation into different species.

\section{References}

[1] Anon (1968). The Preparation of Wood for Microscopic Examination. Forest Products Research Laboratory Leaflet No. 40. Ministry of Technology London, pp 11

[2] Huxley A. (1992). New RHS Dictionary of Gardening 2: 96-97.

[3] Pandey B.P. (2004). Plant Anatomy. S. Chand and Company Ltd, Ram Nagar, New Delhi, pp. 109-136.

[4] Pires J. C., Maureira I. J., Rebman J. P., Salazar G. A., Cabrera L. I., Fay M. F. and Chase M. W.(2004). "Molecular Data Confirms the Phylogenetic Placement of the Enigmatic Hesperovallis (Hesperocallidaceae) with Agave". Madrono 51: 307-311.

[5] Waterhouse J. T. (1987). The Phylogenetic Significance of Dracaenatype Growth. Proc. Linn. Soc. N. S. W.109: 129-138. 